Appl. Phys. B 50, 35-38 (1990)

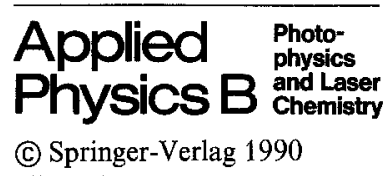

\title{
Laser Evaporation as a Source of Small Free Radicals
}

\author{
M. Ebben, G. Meijer, and J. J. ter Meulen \\ Department of Molecular and Laser Physics, Katholieke Universiteit Nijmegen, \\ Toernooiveld, NL-6525 ED Nijmegen, The Netherlands
}

Received 20 June 1989/Accepted 8 September 1989

\begin{abstract}
It is shown that laser evaporation of a solid target followed by adiabatic expansion can be used to produce cold beams of neutral small open shell molecules. LIF is applied to detect $\mathrm{CuH}\left(X^{1} \Sigma\right), \mathrm{CH}\left(X^{2} \Pi\right), \mathrm{SiH}\left(X^{2} \Pi\right), \mathrm{CuO}\left(X^{2} \Pi\right)$, and $\mathrm{FeO}\left(X^{5} \Delta\right)$. A production of $10^{9 \pm 1} \mathrm{molecules} / \mathrm{sr}$ in a single pulse is obtained for $\mathrm{SiH}$. For a copper target, the optical emission of the jet plume is discussed.
\end{abstract}

PACS: $35.80 .+\mathrm{s}$

Small molecular radicals play an important role as intermediate products in chemical reactions. The study of their electronic structure and therefore their electronic spectra is of fundamental interest in order to understand the chemical processes involving these species. In general spectroscopic experiments on free radicals suffer from low concentrations due to their chemical instability. In the laboratory these species are usually produced in high temperature sources like arcs, ovens, exploding wires, flames and discharges which all have in common that the temperatures (translational, vibrational and rotational) of the resulting products are consequently high. This results in many occupied rovibrational levels in the electronic ground state which in turn gives rise to congested spectra that are generally too complicated to be unraveled completely.

Laser evaporation in combination with supersonic expansion has been shown to produce rotationally cold beams of species, normally to be found only in very hot environments [1-4]. In this way even the most refractory materials can be evaporated and expanded to produce atoms, molecules and clusters, both neutral and ionic, from dimers to droplets.

The processes to be controlled that influence the formation of the different products, are the heating of the plasma that is formed immediately after the laser ablation and the subsequent cooling of these products in the supersonic expansion. Several groups have focused their attention on the production of beams of larger metal clusters and cluster ions that were analysed by mass-spectrometric techniques. In the present experiment we investigated whether this source is suitable for the production of high densities of small free radicals in a molecular beam. For this purpose we aimed at diatomics in the first place formed from one atom from the solid and one atom from a gas added to the seeding gas, if not the seeding gas itself. In various cluster experiments [5-7] diatomic oxides were seen to be present in the beam. In a paper of Simard et al. [8] an experiment is described in which a low concentration of oxygen in the helium carrier gas led to the production of the oxyde of zirconium. In an experiment by Walkup et al. [9] LIF was used to show the presence of several diatomic products in plasma sputtering and reactive ion etching. There were indications that it should be possible to produce a wide variety of small molecules with a laser generated plasma.

\section{Experimental}

The nozzle part of our experimental setup is similar to the one used by Smalley and coworkers $[1,3]$ in their studies of metal cluster beams. Whereas in these experiments several types of extensions of the flow channel are used to increase the yield of larger clusters in the beam (see for example [10]), we deliberately designed the channel to be as short as possible in order to prevent the products from clustering and to avoid 
secondary reactions as much as possible. The channel in which the evaporation takes place is schematically depicted in Fig. 1. In this diagram, all dimensions are drawn to scale. The pulsed valve, a modified version of a Bosch fuel injection valve, provides $100 \mu$ s FWHM gas pulses. An electromagnet opens the $1 \mathrm{~mm}$ diameter nozzle. It is closed by a mechanical spring. The duration of the current through the coil in combination with the tension in the spring, critically determines the temporal evolution of the density in the gas pulse, which is monitored $200 \mathrm{~mm}$ downstream from the nozzle by means of a conventional vacuum ionisation gauge. Gas stagnation pressures of $1-4$ bar are applied. At maximum gas density in the $10 \mathrm{~mm}$ long and $2 \mathrm{~mm}$ wide channel, $1-10 \mathrm{~mJ}$ of third harmonic radiation $(355 \mathrm{~nm})$ from a $Q$-switched Nd:YAG laser (Quanta Ray DCR2) is focused onto the outer surface of a solid target disc (6 $\mathrm{mm}$ thick and $50 \mathrm{~mm}$ diameter) by a $50 \mathrm{~cm}$ focal length lens (estimated spot diameter: $0.2 \mathrm{~mm}$ ). During operation, the solid disc is rotated and translated continuously to achieve maximum shot to shot stability of the density of products in the molecular jet by preventing the Nd:YAG laser from drilling deep holes into the material. In this way a target disc surface can be used for a period of roughly $10 \mathrm{~h}$, without significant decrease of beam quality. The plasma formed during the laser pulse is cooled by the adiabatic expansion into the vacuum chamber. Between two shots, the vacuum recovers to $10^{-5}$ mbar. At a distance of $10 \mathrm{~mm}$ downstream from the nozzle opening and after an appropriate time delay the jet is crossed perpendicularly with the beam of an excimer

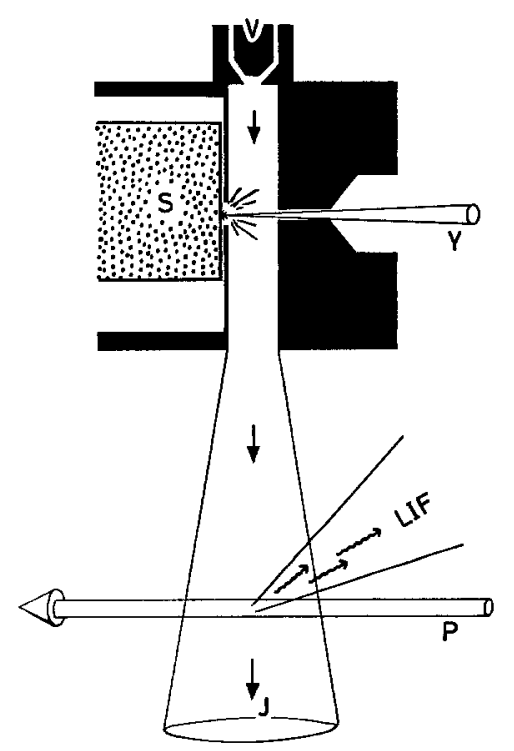

Fig. 1. The evaporation channel. The dimensions are drawn to scale. V: pulsed valve, S: solid, Y: beam of Nd:YAG laser, J: jet, $\mathrm{P}$ : probe laser, LIF: laser induced fluorescence laser pumped dye laser (Lambda Physik EMG 201 $+\mathrm{Fl}$ 2002). The bandwidth of the laser radiation was $0.2 \mathrm{~cm}^{-1}$. The LIF is collected by a short focal length condensor and, after spatial filtering, imaged on the cathode of a photomultiplier tube (EMI 9863). Several optical bandpass and cutoff filters were used to suppress the emission of the jet. The resulting signal is fed into a digital oscilloscope (LeCroy 9400) and a gated integrator (SRS 250), respectively, to allow for time and spectrally resolved scans simultaneously. The repetition rate of the experiment is $10 \mathrm{~Hz}$ and a typical time constant for the gated integrator is $1 \mathrm{~s}$.

\section{Results and Discussion}

A schematic time diagram of the light detected by the photomultiplier is shown in Fig. 2. At time $t=0$ the $\mathrm{Nd}$ :YAG laser is fired. After a short time of flight a beam emission, exhibiting typical photon noise (not shown in the diagram) is seen. This emission is interpreted to be caused by locally produced highly excited atoms that pass through the detection volume, analogous to the $\mathrm{C}_{2}$ excitation in a similar evaporation experiment by Anselment et al. [11]. The shape of the emission curve simply reflects the sensitivity distribution of the imaging optics over the detection volume multiplied by the temporal distribution of the density of emitting atoms in this volume. Obviously, the intensity of this emission strongly depends on the material evaporated and the typical time of arrival differs for different seeding gases. The radicals detected in the beams were all found at the time of maximum beam emission. By scanning the delay time between the $\mathrm{Nd}$ :YAG laser and the probe laser over a large range, also Mie scattering from macroscopically large particles was observed after times of the order of $100 \mu \mathrm{s}$.

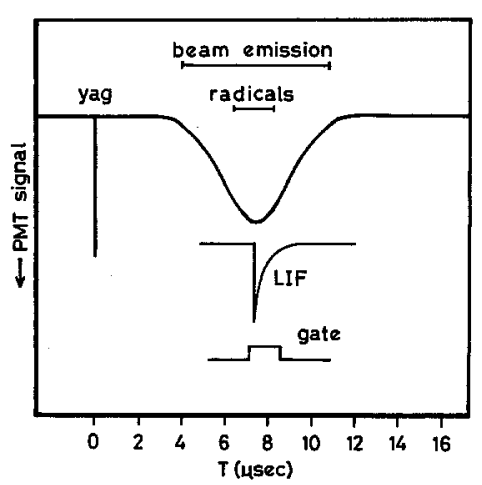

Fig. 2. Schematic time diagram of the photomultiplier signals and the detection gate. The shape of the beam emission reflects the sensitivity volume of the imaging optics. Radical signal was only obtained in the central region of this emission 
This clearly demonstrates that although we used high energy $(355 \mathrm{~nm})$ photons for evaporation and heating up the plasma in combination with a short expansion channel in order to preferentially produce small compounds $[12,13]$, big clusters are also formed and observed after appropriate delay times. The presence of these large particles in the beam is in good agreement with the Planck emission study of Rohlfing [14]. In accordance with many cluster-ion experiments, an impressive flow of ions was found to be produced.

$\mathrm{CuH}$ was choosen as the first candidate because it is a closed shell molecule and thus most likely to survive, if produced, the first high pressure stage of the expansion. Probing the well known [15] $A^{1} \Sigma^{+}\left(v^{\prime}=0\right) \leftarrow X^{1} \Sigma^{+} \quad\left(v^{\prime \prime}=0\right) \quad$ transition around $429 \mathrm{~nm}$ yielded the spectrum of which a part is shown in Fig. 5. The rotational temperature was determined to be $45 \mathrm{~K}$, if 1 bar of pure hydrogen is used as seeding gas.

In the case of copper as target material, the beam emission was particularly intense in the green as could readily be observed with the naked eye. This emission extended over a distance of $50 \mathrm{~mm}$ from the channel orifice. On dispersion it was shown to originate from cascade decay involving the allowed transitions in the copper atom from states with energies just below the ionisation limit down to the ground state. In order to investigate the origin of these $\mathrm{Cu}^{*}$ atoms we applied an electric field $(0-3000 \mathrm{~V} / \mathrm{m})$ both parallel and antiparallel to the jet. The parallel field did not influence the emission at all, while the antiparallel field shortened the range of green emission in proportion to the applied field strength. This led us to the conclusion that positive (cluster-) ions are involved in this $\mathrm{Cu}^{*}$ formation while low energy electrons cannot be. This rules out the recombination of $\mathrm{Cu}^{+}$with a low energy electron as well as the collisional dissociation of larger clusters induced by such electrons as proposed by Anselment et al. [11] for the electronically excited $C_{2}$ they observed. As the pressure in the detection volume is estimated to be of the order of 1 Torr, i.e. rather high, the $\mathrm{Cu}^{*}$ formation could well be a result of collisional fragmentation of larger positive clusters by neutral species. The exact nature of this $\mathrm{Cu}^{*}$ formation process, however, is still unclear.

$\mathrm{SiH}$ was the first actual radical we investigated. For this purpose pure silicon was evaporated and entrained in hydrogen. The probe laser was tuned to the $A^{2} \Delta\left(v^{\prime}=0\right) \leftarrow X^{2} \Pi \quad\left(v^{\prime \prime}=0\right)$ transition in $\mathrm{SiH}$ at $412 \mathrm{~nm}$. The resulting spectrum is shown in Fig. 3. For the assignment of the spectrum we used the data from the work of Klynning et al. [16]. The rotational temperature was determined to be $30 \mathrm{~K}$. From the time resolved fluorescence signal resulting from the excitation of molecules in a single rotational state, we calculated the absolute number of $\mathrm{SiH}$ molecules produced in this way to amount $10^{9 \pm 1}$ molecules/sr in a single pulse.

Besides pure hydrogen, mixtures of different percentages of oxygen in helium were also applied as carrier gas. In this way we produced $\mathrm{CuO}\left(X^{2} \Pi\right)$ and $\mathrm{FeO}\left(X^{5} \Delta\right)$ in large amounts as inferred from their LIF spectra $[17,18]$. As an example, the spectrum of the $A^{2} \Sigma^{+}\left(v^{\prime}=0\right) \leftarrow X^{2} \Pi_{3 / 2}\left(v^{\prime \prime}=0\right)$ transition of $\mathrm{CuO}$ is shown in Fig. 4. A computer simulation of the low temperature spectrum of this transition, using the molecular parameters as determined by Appelblad and Lagerqvist [17] from their high temperature spectra, yielded a rotational temperature of $60 \mathrm{~K}$, still moderately high. It should be noted however that our spectra were measured at a distance of $10 \mathrm{~mm}$ downstream from the $2 \mathrm{~mm}$ outlet opening of the expansion channel which means still in the middle part of the jet expansion, where the temperatures have not yet reached their final values. We wish to emphasize that these open shell radicals survive the expansion.

Figure 5 shows the spectra of both the $A^{1} \Sigma^{+}\left(v^{\prime}=0\right) \leftarrow X^{1} \Sigma^{+}\left(v^{\prime \prime}=0\right)$ transition in $\mathrm{CuH}$ and the $A^{2} A\left(v^{\prime}=0\right) \leftarrow X^{2} \Pi\left(v^{\prime \prime}=0\right)$ transition in $\mathrm{CH}$ [19], excited in the same frequency region. These spectra occurred simultaneously when a copper disc, hosting an unknown small concentration of carbon was

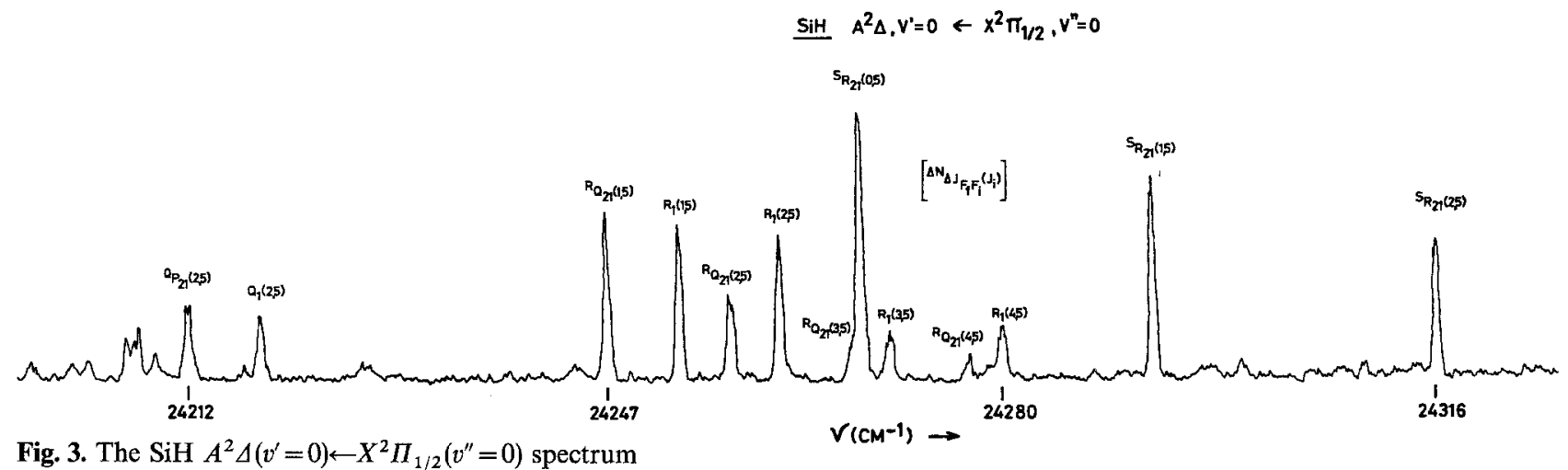

Fig. 3. The $\mathrm{SiH} A^{2} \Delta\left(v^{\prime}=0\right) \leftarrow X^{2} \Pi_{1 / 2}\left(v^{\prime \prime}=0\right)$ spectrum 
CuO $\quad A^{2} \Sigma^{4}\left(v^{\prime}=0\right) \leftarrow x^{2} \pi_{3 / 2}(v=0)$

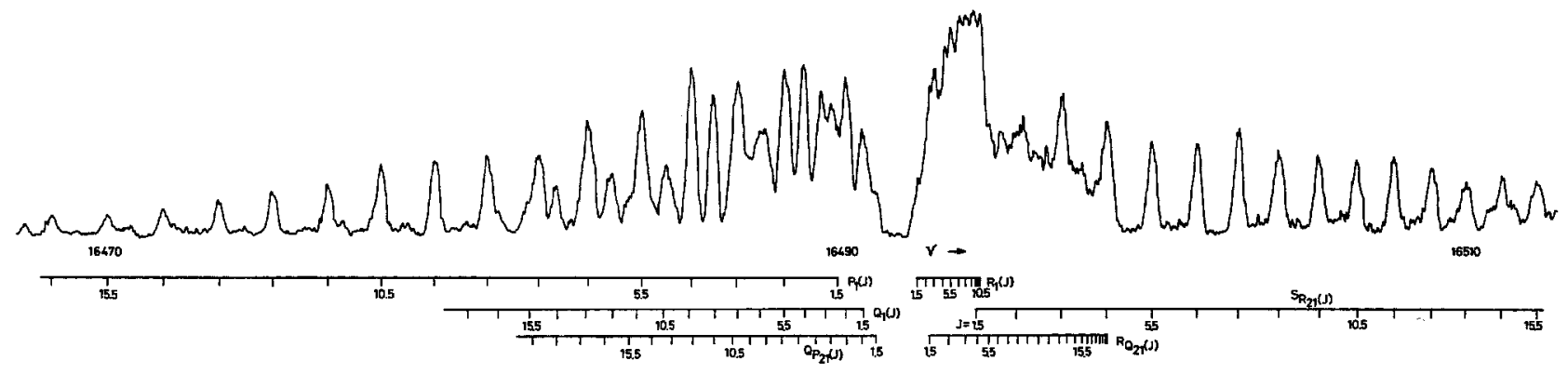

Fig. 4. The $\mathrm{CuO} A^{2} \Sigma^{+}\left(v^{\prime}=0\right) \leftarrow X^{2} \Pi_{3 / 2}\left(v^{\prime \prime}=0\right)$ spectrum. The $\mathrm{R} 1$ head is truncated due to photomultiplier saturation

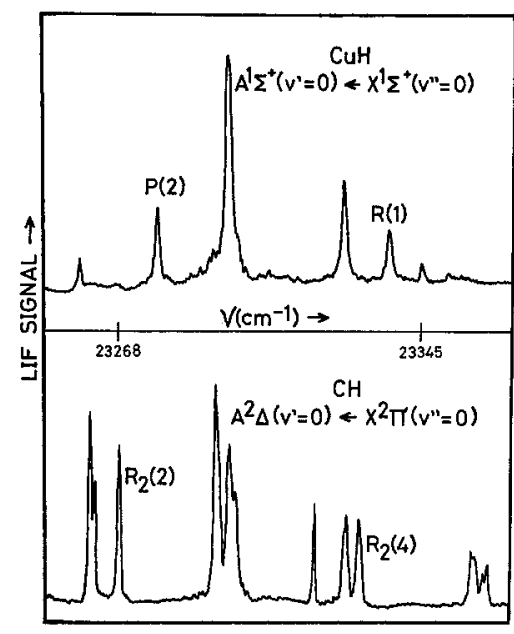

Fig. 5. The $\mathrm{CuH} A^{1} \Sigma^{+}\left(v^{\prime}=0\right) \leftarrow X^{1} \Sigma^{+}\left(v^{\prime \prime}=0\right)$ spectrum and the $\mathrm{CH} A^{2} \Delta\left(v^{\prime}=0\right) \leftarrow X^{2} \Pi\left(v^{\prime \prime}=0\right)$ spectrum. The spectra are recorded under identical conditions, the only difference being the position of the boxcar gate, from 0 to $300 \mathrm{~ns}$ and from 200 to 500 ns after the probe laser pulse for $\mathrm{CuH}$ and $\mathrm{CH}$ respectively

irradiated in the presence of hydrogen. The spectra were unraveled by simply increasing the time delay between the probe laser and the gate of the boxcar averager, thus probing molecular states with shorter $(\mathrm{CuH}: 200 \mathrm{~ns})$ and longer (CH: $530 \mathrm{~ns})$ radiative lifetimes respectively. The fact that traces of carbon in the solid target give rise to a high density of $\mathrm{CH}$ in the beam indicates that the method presented in this paper can also be applied using alternative sources of atoms, for example, thin layers, alloys or deposited salts, clearly depending on the molecule under investigation.

Work is underway to apply the method presented to molecules that are hard to produce and possess unknown electronic structures. We plan to apply a single frequency $\mathrm{cw}$ dye laser in order to obtain high resolution spectra and to investigate highly excited electronic states by pulsed-cw double resonance [20].
Acknowledgements. We wish to thank E. van Leeuwen, Drs. R. Spooren, R. van Dijck, and Drs. M. Pieksma for their expert help in the construction of the apparatus. We thank Prof. J. Reuss for stimulating discussions.

\section{References}

1. R.E. Smalley: Laser Chem. 2, 167 (1983)

2. Heon Kang, J.L. Beauchamp: J. Phys. Chem. 89, 3364 (1985)

3. D.E. Powers, S.G. Hansen, M.E. Geusic, D.L. Michalopoulos, R.E. Smalley: J. Chem. Phys. 78, 2866 (1983)

4. J.L. Gole, J.H. English, V.E. Bondeybey: J. Phys. Chem. 86, 2560 (1982)

5. E.A. Rohlfing, D.M. Cox, A. Kaldor: J. Phys. Chem. 88, 4497 (1984)

6. D.W. Powers, S.G. Hansen, M.E. Geusic, A.C. Pulu, J.B. Hopkins, T.G. Dietz, P.R.R. Langridge-Smith, R.E. Smalley: J. Phys. Chem. 86, 2556 (1982)

7. E.A. Rohlfing, D.M. Cox, A. Kaldor: Chem. Phys. Lett. 99, 161 (1983)

8. B. Simard, S.A. Mitchell, M.R. Humphries, P.A. Hackett: J. Molecular Spectroscopy 129, 186 (1988)

9. R. Walkup, P. Avouris, R.W. Dreyfus, J.M. Jasinsky, G.S. Selwyn: Appl. Phys. Lett. 45, 372 (1984)

10. Y. Liu, Q.L. Zhang, F.K. Tittel, R.F. Curl, R.E. Smalley: J. Chem. Phys. 85, 7434 (1986)

11. M. Anselment, R.S. Smith, E. Daykin, L.F. Dimauro: Chem. Phys. Lett. 134, 444 (1987)

12. M.E. Geusic, M.D. Morse, S.C. O'Brien, R.E. Smalley: Rev. Sci. Instrum. 56, 2123 (1985)

13. M. Costes, C. Naulin, G. Dorthe, C. Vaucamps, G. Nouchi: Faraday Discuss. Chem. Soc. 84, 75 (1987)

14. E.A. Rohlfing: J. Chem. Phys. 89, 6103 (1988)

15. A. Heimer, T. Heimer: Z. Physik 84, 222 (1933)

16. L. Klynning, B. Lindgren, U. Sassenberg: Physica Scripta 20, 617 (1979)

17. O. Appelblad, A. Lagerqvist: Physica Scripta 10, 307 (1974)

18. A.S.C. Chueng, A.M. Lyyra, A.J. Merer, W. Taylor: J. Mol. Spectrosc. 102, 224 (1983)

19. S.V. Filseth, H. Zacharias, J. Danon, R. Wallenstein, K.H. Welge: Chem. Phys. Lett. 58, 140 (1978)

20. Gerard Meijer, Maarten Ebben, J.J. ter Meulen: Chem. Phys. 127, 173 (1988) 